\title{
Designing an optical frequency comb generator for visible light communication applications
}

\author{
Marwa Jaleel Mohsin ${ }^{1,2}$, Ibrahim A. Murdas ${ }^{1}$ \\ ${ }^{1}$ Electrical Engineering Department, College of Engineering, University of Babylon, Hillah, Iraq \\ ${ }^{2}$ Engineering Technical College, Al-Furat Al-Awsat Technical University, Al-Najaf, Iraq
}

\begin{tabular}{l} 
Article Info \\
\hline Article history: \\
Received Apr 8, 2021 \\
Revised Jul 15, 2021 \\
Accepted Aug 2, 2021 \\
\hline Keywords: \\
Amplitude modulation \\
Mach-Zehnder modulator \\
OFCG \\
Power fluctuation \\
VLC
\end{tabular}

\section{Article Info}

Article history:

Received Apr 8, 2021

Revised Jul 15, 2021

Accepted Aug 2, 2021

\begin{abstract}
The optical frequency comb generator (OFCG) is an efficient optoelectronic device that is included in many important applications over a various field such as microwave and optical communication. A novel scheme of OFCG presented in this work for visible light communication application based on amplitude modulation, radio frequency (RF) signal, phase shift and two Mach-Zehnder modulators (MZMs), our design features are simple with more efficient power and premium flatness of comb lines, the number of generating frequencies lines was 64 with a power stronger than $-2 \mathrm{dBm}$ over a $340 \mathrm{GHz}$ bandwidth from a single continuous laser diode. Different chirping factor $(\alpha)$ of MZMs are implemented $(3,5,7)$, as the results the best results related to $\alpha=5$ with extra flatness, the system was designed and simulated by VPI design suite 9.8 .
\end{abstract}

This is an open access article under the CC BY-SA license.

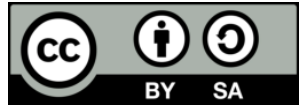

\section{Corresponding Author:}

Marwa Jaleel Mohsin

Electrical Engineering Department, College of Engineering, University of Babylon

Hillah, Iraq

E-mail: marwa.jaleel@atu.edu.iq

\section{INTRODUCTION}

Optical frequency comb generator (OFCG) is a vital component source for many applications based on multi-wavelength sources such as optical frequency measurement, optical frequency division multiplexing (OFDM), microwave photonic signal processing, optical arbitrary waveform generation and dense wavelength division multiplexing [1]-[3]. the basic principle of OFCG is to generate a set of optical signals or harmonics from a single optical source with maximum exploitation of the optical bandwidth, the spacing between these harmonics is tunable [4], [5]. Can benefit of OFCG by reducing the optical sources in the optical line terminal (OLT) side with providing broad bandwidth and high transmission data rate [6].

The key characteristics of OFCG are equal frequency spacing between lines, optical spectrum flatness, the narrow linewidth of each comb line, low implementation cost and wide spectrum wavelength range [2], [6], [7]. Many different techniques were presented by many researchers over the last years each one has its merits and demerits, these techniques were categorised into two methods, electrical and optical, the electrical one was limited by the bandwidth of the electrical components while the optical method allowed broader bandwidth, therefore the optical method is the most prevalent in the researches nowadays [7], [8]. For the optical method, one technique to implementation OFCG by using mode-locked laser (MLL) such as in [8]-[10], the mean disadvantages of this method are the stability and its hard to tunable the spacing between lines, because of the carrier frequency depending on the cavity of the laser diode due to the environments, for stable operation, it required a complicated feedback loop for stable operation [11], [12].

Another technique is based on fiber nonlinearity, such as the four-wave mixing effect (FWM) and stimulated brillouin scattering (SBS), this technique characterized by complex structure and required high 
power optical amplifiers and optical filter to sort the optical spectrum [1]-[3], [7], [13]. The most common and efficient technique is the optical modulation technique, which's based on optical modulation of the narrow internal or external seed source to produce multiple wavelengths or frequency comb lines by using single or multiple modulators with nested, cascaded or loop structures [6]. This method characterized by stability easy tunable comb spacing and flexibility [1], [14], [15].

Modulating the continuous wave $(\mathrm{CW})$ diode can be performed using a single modulator, the system will be simple and generated a limited number of comb lines [10], [11], [16]. To increase the number of comb line, cascaded scheme of modulator were presented. This technique restricted by the insertion losses of the optical modulator which limits the bandwidth [6], [17].

Another important technique is ring or loop structure, called recirculating frequency shifting (RFS). This technique is more efficient than modulation technique in the term of bandwidth [6], [18]-[20], it implemented by different ways in many researchers as explained in next section. In this work, we proposed a novel scheme for visible light communication (VLC) application such as light fidelity (Li-Fi) based on CW laser, amplitude modulation (AM), two Mach-Zehnder modulators, phase shift. The chirping factor of MachZehnder modulators (MZMs) was varied with three different values $(\alpha=3,5,7)$ and compare the results to study the effect of the chirping factor on the flatness of the comb generator output. We obtained 64 comb line with very good optical power and an excellent level of flatness over $340 \mathrm{GHz}$ bandwidth.

\section{LETRECHER REVIEW}

We classified the previous works based on the techniques that were used to implemented OFCG. For the MLL technique, the centre frequency is fixed by the design of laser and spaced comb line determined by laser cavity length [6], [21]. Mode locked laser diode (MLLD) structure can be linear or ring cavity structures. Harvey and Mollenauer [12], Shan and Spirit [13], and Kato et al. [14] employ loop of optical fiber and comb generator equipped with a Fabry-Perot light modulator, in this method the frequency intervals are precisely constant and fixed phase rotation between spectrum components. This method is unstable due to fluctuation of the path so this method required a more complicated stabilization system [15]. Kawanishi et al. [15] designed OFCG based on loop system fabricated from mode-locked ring laser, multi-project wafer run in an active and passive integrated process using multimode interference (MMI), semiconductor optical amplifier (SOA), electro-optic phase modulator, SA and passive waveguide, the best results of this work were 44 lines with flatness $1.8 \mathrm{dBm}$ variation with maximum output power about $-18 \mathrm{dBm}$. Shen et al. [16] designed OFCG span over $2 \mathrm{THz}$ contain 200 optical comb line stronger than $-20 \mathrm{dBm}$ using fiber loop, optical phase modulator and erbium doped fiber amplifiers (EDFA).

For cascade technique, it adopted in [1]-[5], [7], [17]. Qu et al. [1] proposed a novel scheme to generate flatter and stable OFC based on chirping of two stages MZM, the first stage used dual-drive MZM with high chirp factor and for increasing the comb lines used integrated MZM in the second stage. The chirp factor is changed $(\alpha=5,7$ and 9), the number of comb line was in the range between 15 to 21 with output power is about $-20 \mathrm{dBm}$. Hmood et al. [2] designed a stable and flat OFCG based on MZMs with high chirping factor by two different methods, in the first one the electrical RF signal modulated the optical signal using a single MZM while the second used two cascades MZMs and time delay, the number of achieved comb lines was 13 with power fluctuation less than $1 \mathrm{~dB}$ and power strength about $-10 \mathrm{dBm}$. Zhang et al. [3] proposed a FCG based on two stages of polarization modulators (PolMs) and MZM. The first stage contains PolM and MZM produced $11 \mathrm{comb}$ line with good flatness followed by the second stage which contains another PolM this will increase the comb lines by a factor of up to 5. The system is cost-effective. The frequency spacing between comb line was $5 \mathrm{GHz}$ while the amplitude of RF signal is changed between three value, the maximum number of comb lines was 55 lines with fluctuation power $5.24 \mathrm{dBm}$ and output power about $-20 \mathrm{dBm}$. Ferreira and Rocha [4] presented a theoretical study with three techniques for implementing comb generator based on cascade model of modulator, RFS and discrete mode laser, stimulated the proposed design by optiSystem and compered between them in the term of bit error rate (BER). Ullah et al. [5] generating $60 \mathrm{comb}$ lines with good power fluctuation about $0.7 \mathrm{dBm}$ over $0.5 \mathrm{THz}$ bandwidth based on a cascade module of pulsed laser diode, phase modulator and two MZMs. All modulators are driven by the same RF signal. This system was designed for implementing wavelength division multiplex passive optical network (WDM-PON) to support the $1.2 \mathrm{THz}$ transmission data rate. Shen et al. [7] proposed a novel system of microwave frequency generator with Alta-flat broadband based. The system was easy to adjust and simple. The proposed scheme based on optical frequency comb (OFC) with multiple-quantum-well electroabsorption modulator (MQW-EAM) and contain top level domain (TLD) with EDFA. The spacing between lines can be adjusted by changing RF signal frequency from 5 to $20 \mathrm{GHz}$, the results show 15 comb lines with maximum output power is $-30 \mathrm{dBm}$ over $300 \mathrm{GHz}$ effective bandwidth and $0.02 \mathrm{dBm}$ power fluctuation. Ullah et al. [17] proposed a cascade module of three modulators, one amplitude modulation (AM) and two 
MZMs with a laser diode to achieve a novel and effective frequency comb generator (FCG), RF signal of $30 \mathrm{GHz}$ used to drive the three modulators and it is the spacing between the comb lines, number of flat comb lines generated was 61 with $40 \mathrm{~dB}$ TNR.

\section{BASIC PRINCIPLE}

OFCG as mention before using to generating a set of optical lines over a large bandwidth from the same laser source that will reduce the optical sources in the optical line terminal (OLT) and increase the transmission data rate of the system due to the multi carriers [17], [22], [23]. The proposed system was generating a set of optical comb lines for visible light communication $(400-800 \mathrm{THz})$ and dense wavelength division multiplexing (DWDM) applications. The proposed as shown in Figure 1.

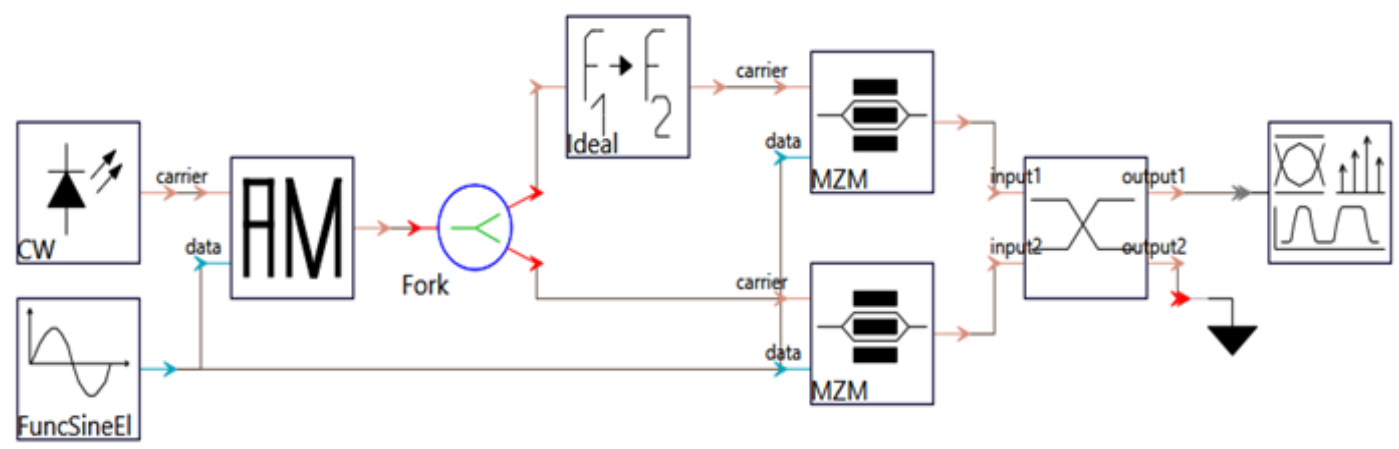

Figure 1. The proposing scheme of OFCG

The CW laser source was emitted at frequency $500 \mathrm{THz}$, the light of the laser source with frequency $\mathrm{f}_{0}$ has an amplitude $A_{0}$ and phase $\varphi_{0}$. The probability density function is used to moduled the laser phase noise as:

$$
f(\Delta \varphi)=\frac{1}{2 \pi \sqrt{\Delta f d t}} \cdot e^{-\Delta \varphi^{2} / 4 \pi \Delta f d t}
$$

where $\Delta \varphi$ is the phase difference between two consecutive time instances and $\Delta f$ is the line width of laser diode, between two successive time instance a summed a Gaussian random variable with zero mean and variance about $2 \pi \Delta f$. A sequence of harmonic tones with $f_{0}+n f$ components will be on the output of the amplitude modulator, where $f$ is the RF signal frequency while $n=[0,1,2, \ldots]$ is the number of the harmonics. The output field of AM is given by [17], [24]:

$$
E_{k}=\left|E_{0}\right| \sum_{n=\infty}^{-\infty} E_{n, k} \cos \left[2 \pi\left(f_{0}+n f\right) t+\theta_{n, k}\right.
$$

$E_{n, k}$ and $\theta_{n, k}$ are the amplitudes and phases of component respectively [17]. The output of the amplitude modulator was driven into two branches one will be applied to the MZM2 and the other one will be shifted by $f_{0}+n_{\max } f$ and applied to MZM1. The modulators are driven by RF signal, the spacing of the generator comb lines can be tunable by RF signal frequency. All the modulators are driven by the same RF signal. The MZM have an output optical power depends on the $\Delta \emptyset$ of the two brunches [19], [25]:

$$
P_{\text {out }}(t)=P_{\text {in }}(t) \cdot d(t)=P_{\text {in }}(t) \cos ^{2}[\Delta \emptyset(t)]
$$

with

$$
\Delta \emptyset(t)=\frac{\Delta \emptyset_{1}(t)-\Delta \emptyset_{2}(t)}{2}
$$

$d(t)$ is the power transfer function while phase change in each brunch is $\Delta \emptyset_{1}(t)$ and $\Delta \emptyset_{2}(t)$ which is caused by applied signal. 


\section{RESEARCH METHOD}

The proposed system for generating optical comb lines for visible light communication and DWDM application as shown in Figure 1, the CW laser diode was used as an optical source emitted at $500 \mathrm{THz}$. The light of the laser has a spectrum with a single carrier frequency centered at $500 \mathrm{THz}$ and many weak sidebands, applied to AM modulator to produce multi harmonics at the two sides of the center frequency. These harmonics are gradually decreased on both sides, the output of AM was separated into two branches by Fork, one of these directly passed through the $\mathrm{MZM}_{2}$ and the other one shifted by phase shifter then passed through the $\mathrm{MZM}_{1}$, frequency offset of the phase shift is equal to the AM bandwidth this process takes advantageous in double the overall system bandwidth. The output harmonics after $\mathrm{MZM}_{1,2}$ was stronger and flatter, then combined using un optical coupler to produce the final signal of OFCG.

All the modulators were driven from the same FR signal. RF signal frequency is controlling on the duration of comb lined so the spacing between generating optical lines was tunable by RF frequency by increasing the frequency the spacing between optical comb lines will increase. The alpha factor of the MZMs is varied over 3, 5, 7 to experience the best result of OFCG. The proposing OFCG has a very good result in term of flatness and output power compared to the many previous works. We obtained 64 comb lines over $340 \mathrm{GHz}$ bandwidth; the output power reaches to $8 \mathrm{dBm}$.

\section{RESULTS AND DISCUSSION}

The proposed system was stimulated using VPIphotonic design suite 9.8, all the parameters were used for designing the system was explained in Table 1. Figures 2(a), (b) show the output of the CW laser diode and the AM modulator. AM produces multiple harmonics of the input signal on the two sides of CW center frequency of optical spectrum after passed this signal through the two parallel MZMs these harmonics will be stronger and flatter, the value of alpha-factor affects the flatness of these harmonics as demonstrated in Figures 3(a), (b), (c).

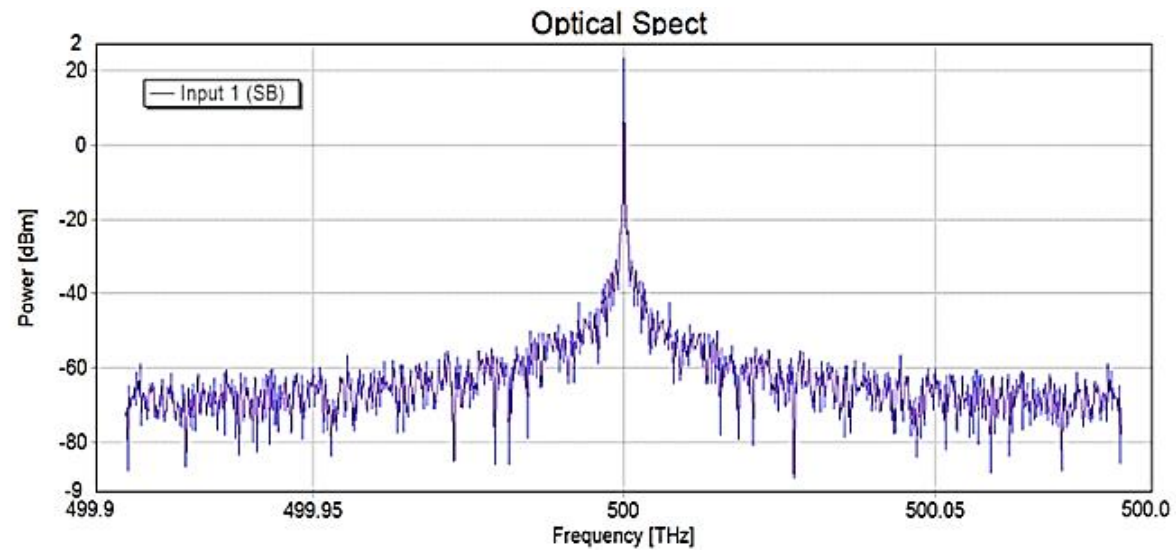

(a)

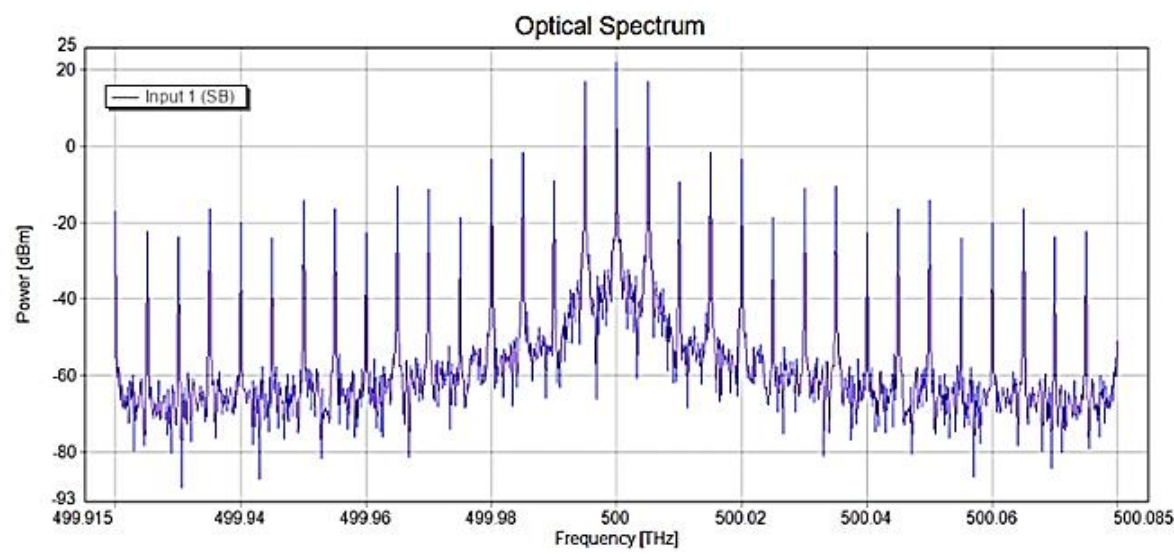

(b)

Figure 2. Output optical spectrum of (a) CW laser, (b) AM modulator 
As the results, we obtained 64 comb lines for VLC frequencies with spacing is $5 \mathrm{GHz}$ this spacing easy tunable by FR signal frequency, the bandwidth of the proposed system is $340 \mathrm{GHz}$, the Figure 3 shows the output of the proposing OFCG with three different values of alpha-factor, the best results belong to the value 5 of an Alpha factor due to the high flatness. Table 2 explains the measured parameter of stimulated OFCG, the best results related to $\alpha=5$ with excellent flatness with strong output power. As shown in Figure 3(b), the overall comb lines results is very good compared with mentioned previous work in section 2 , there are 42 smooth and strong comb lines with power fluctuation $0.5 \mathrm{dBm}$, and the other comb line ranges between $-1.77 \mathrm{dBm}$ and $-2.14 \mathrm{dBm}$.

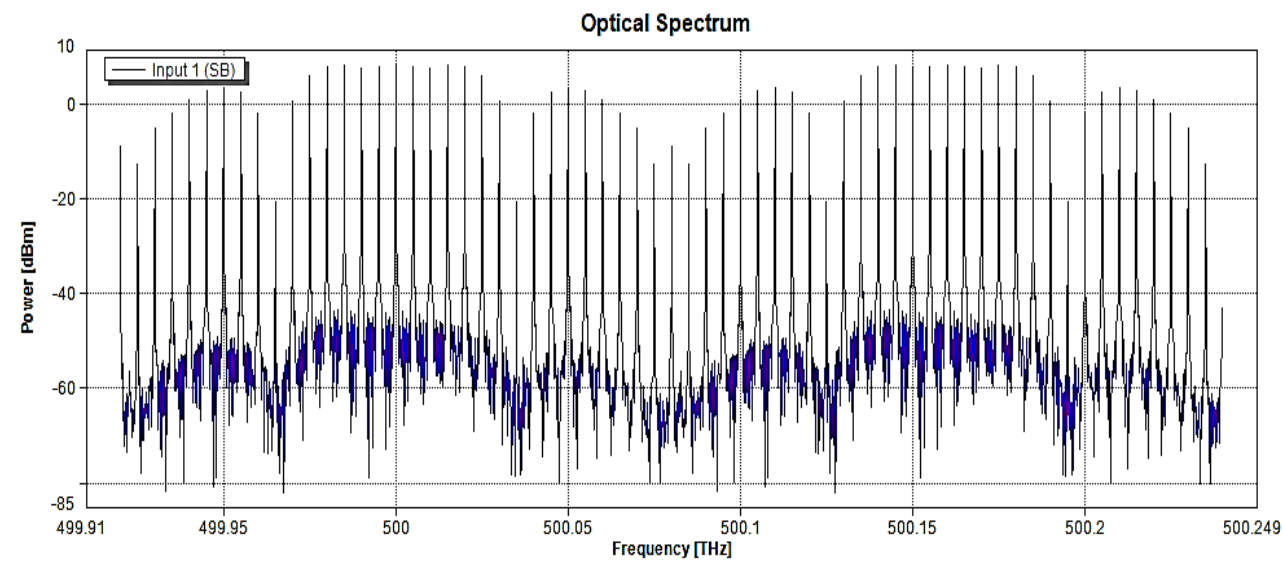

(a)

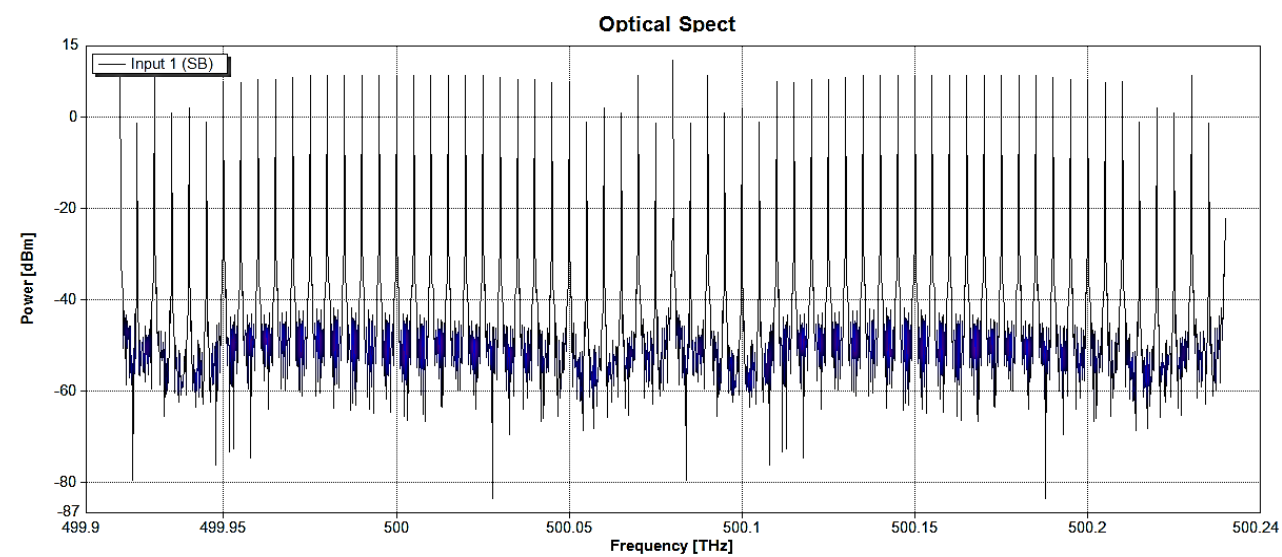

(b)

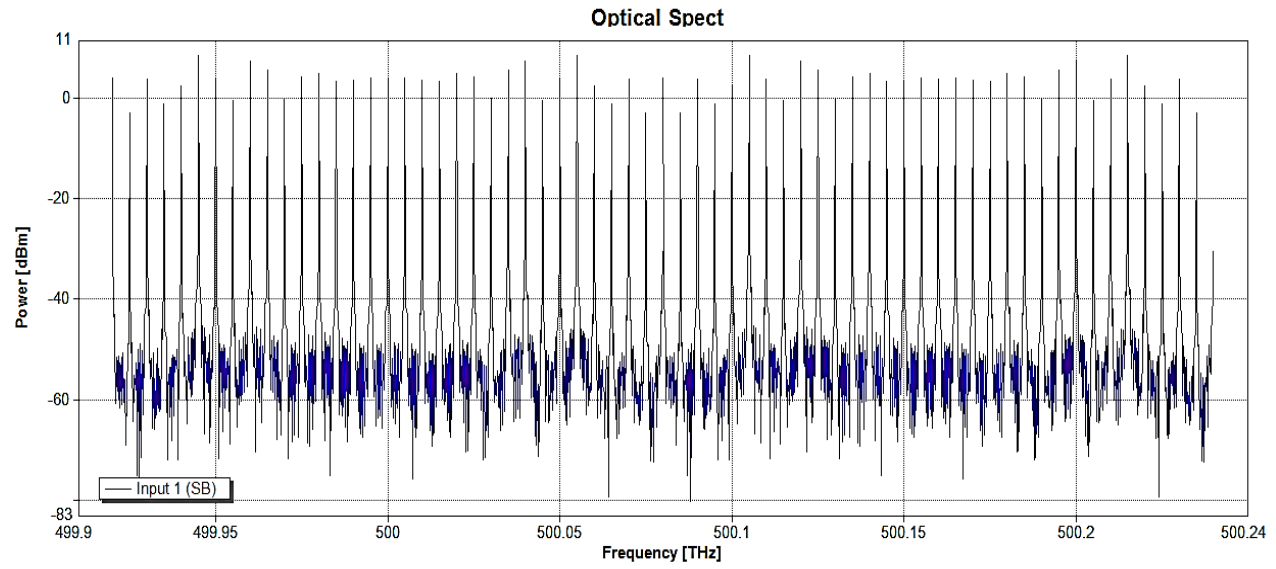

(c)

Figure 3. The generation of OFC lines with: (a) $\alpha=3$, (b) $\alpha=5$, (c) $\alpha=7$ 
Table 1. The parameters of proposed OFCG

\begin{tabular}{ccc}
\hline The Device & Parameter & Value \\
\hline CW Laser & Emission frequency & $500 \mathrm{THz}$ \\
& Average power & $0.2 \mathrm{~W}$ \\
& Linewidth & $100 \mathrm{KHz}$ \\
& Azimuth & $45 \mathrm{deg}$ \\
AM Modulator & Modulation index & 1 \\
Phase Shirt & Frequency offset & $160 \mathrm{GHz}$ \\
RF Signal & Frequency & $5 \mathrm{GHz}$ \\
& Amplitude & $2 \mathrm{a} . \mathrm{u}$. \\
& Bias & $1 \mathrm{a} . \mathrm{u}$. \\
MZMs & Extinction ratio & $35 \mathrm{~dB}$ \\
& Alpha factor & $3,5,7$ \\
\hline
\end{tabular}

Table 2. The stimulated results

\begin{tabular}{ccc}
\hline Alpha Factor & Maximum Output Power $(\mathrm{dBm})$ & Minimum \\
\hline$\alpha=3$ & 8.83 & -20.41 \\
$\alpha=5$ & 8.09 & -2.14 \\
$\alpha=7$ & 10.10 & -2.39 \\
\hline
\end{tabular}

\section{CONCLUSION}

We designed an efficient scheme of optical frequency comb generator (OFCG) based on AM modulator, phase shift and two parallel MZMs for generating a set of carrier frequencies from a single laser source to reduce the optical sources at the transmission side with maximizing the transmission data rate, the system generated comb lines over VLC frequencies and it can be advantageous in the VLC and DWDM applications, and characterized by its simplicity and tunability. The proposed scheme was designed and stimulated by VPIphotonic design suite 9.8. OFCG generates 64 flat comb lines with output power stronger than $-2 \mathrm{dBm}$ over $340 \mathrm{GHz}$ bandwidth, from these lines there is 42 lines with an output power of about 8 $\mathrm{dBm}$ and fluctuation less than $0.5 \mathrm{dBm}$. The spacing between comb line was easily tunable by the RF frequency signal This paper also presented the effect of varying chirping factor of MZMs with three different values $(\alpha=3,5,7)$, the best results related to $\alpha=5$.

\section{REFERENCES}

[1] K. Qu, S. Zhao, D. Y. Liang, Z. Zhu, C. Dong, and X. Li, "High flatness optical frequency comb generator based on the chirping of modulators," Optical Review, vol. 23, pp. 436-441, 2016, doi: 10.1007/s10043-016-0216-8.

[2] J. K. Hmood, S. D. Emami, K. A. Noordin, H. Ahmad, S. W. Harun, and H. M. H. Shalaby, "Optical frequency comb generation based on chirping of Mach-Zehnder Modulators," Optics Communications, vol. 344, pp. 139-146, 2015, doi: 10.1016/j.optcom.2015.01.054.

[3] F. Zhang, X. Ge, and S. Pan, "A two-stage optical frequency comb generator based on polarization modulators and a MachZehnder interferometer," Optics Communications, vol 354, pp. 94-102, 2015, doi: 10.1016/j.optcom.2015.05.068.

[4] R. L. Ferreira and M. L. Rocha, "Optical comb generation techniques," 5th IWT 2013, International Workshop on Telecommunications, 2013.

[5] R. Ullah et al., "Pulsed laser-based optical frequency comb generator for high capacity wavelength division multiplexed passive optical network supporting 1.2 Tbps," Optical Engineering, vol. 55, no. 9, Sep. 2016, Art. no. 096106, doi: 10.1117/1.OE.55.9.096106.

[6] V. Corral, R. Guzmán, C. Gordón, X. J. M. Leijtens, and G. Carpintero, "Optical frequency comb generator based on a monolithically integrated passive mode-locked ring laser with a Mach-Zehnder interferometer," Optics Letters, vol. 41, no. 9, pp. 1937-1940, May 2016, doi: 10.1364/OL.41.001937.

[7] C Shen, X. Zhu, Y. Zhang, and Y. Han, "Ultra-flat broadband microwave frequency comb generation based on optical frequency comb with a multiple-quantumwell electro-absorption modulator in critical state," Fronties of Optoelectronics, vol. 12, pp. 382-391, 2019, doi: 10.1007/s12200-019-0915-4.

[8] N. R. Doloca, K, Meiners-Hagen, M. Wedde, F. Pollinger, and A. Abou-Zeid, "Absolute distance measurement system using a femtosecond laser as a modulator," Measurement Science and Technology, vol. 21, no. 11, 2010, Art. no. 115302, doi: 10.1088/0957-0233/21/11/115302.

[9] K. Madani and C. S. Aitchison, "A $20 \mathrm{GHz}$ microwave sampler," in IEEE Transactions on Microwave Theory and Techniques, vol. 40, no. 10, pp. 1960-1963, Oct. 1992, doi: 10.1109/22.159634.

[10] F. Zhang, J. Wu, Y. Li, and J. Lin, "Flat optical frequency comb generation and its application for optical waveform generation," Optics Communications, vol. 90, no. 1, pp. 37-42, 2013, doi: 10.1016/j.optcom.2012.10.051.

[11] A. J. Metcalf, V. Torres-Company, D. E. Leaird, and A. M. Weiner, "High-power broadly tunable electrooptic frequency comb generator," in IEEE Journal of Selected Topics in Quantum Electronics, vol. 19, no. 6, pp. 231-236, Nov.-Dec. 2013, Art. No. 3500306, doi: 10.1109/JSTQE.2013.2268384.

[12] G. T. Harvey and L. F. Mollenauer, "Harmonically Modelocked fiber ring laser with an internal Fabry-Perot stabilizer for soliton transmission," Optics Letters, vol. 18, no. 2, pp. 107-109, 1993, doi: 10.1364/OL.18.000107.

[13] X. Shan and D. M. Spirit, "Novel method to suppress noise in harmonically modelocked eribum fiber laser," Electronics Letters, vol. 29, no. 11, pp. 979-981, 1993, doi: 10.1049/el:19930652. 
[14] M. Kato, K. Fujiura, and T. Kurihara, "Generation of super-stable $40 \mathrm{GHz}$ pulse from Fabry-Perot resonator integrated with electro-optic phase modulator,” Electronics Letters, vol. 40, pp. 299-301, 2004, doi: 10.1049/el:20040202.

[15] T. Kawanishi, T. Sakamoto, S. Shinada, and M. Izutsu, "Optical frequency comb generator using optical fiber loop with singlesideband modulator," IEICE Electronics Express, vol. 1, no. 8, pp. 217-221, 2004, doi: 10.1587/elex.1.217.

[16] P. Shen, N. J. Gomes, P. A. Davies, and W. P. Shillue, "Generation of $2 \mathrm{THz}$ span optical comb in a tunable fiber ring based optical frequency comb generator," 2007 Interntional Topical Meeting on Microwave Photonics, 2007, pp. 46-49, doi: 10.1109/MWP.2007.4378132.

[17] R. Ullah et al., "Flattened optical multicarrier generation technique for optical line terminal side in next generation WDM-PON supporting high data rate transmission," in IEEE Access, vol. 6, pp. 6183-6193, 2018, doi: 10.1109/ACCESS.2018.2789863.

[18] H. C. Reader, D. F. Williams, P. D. Hale, and T. S. Clement, "Comb-generator characterization," in IEEE Transactions on Microwave Theory and Techniques, vol. 56, no. 2, pp. 515-521, Feb. 2008, doi: 10.1109/TMTT.2007.914630.

[19] A. A. Abdullah and R. S. Fyath, "Towards optical frequency comb-based high-capacity superchannel transmission-part ii: Transmission performance," International Journal of Networks and Communications, vol. 10, no. 2, pp. 33-40, 2020, doi: 10.5923/j.ijnc.20201002.01.

[20] A. K. Mishra et al., "Flexible RF-based comb generator," in IEEE Photonics Technology Letters, vol. 25, no. 7, pp. 701-704, Apr. 2013, doi: 10.1109/LPT.2013.2249509.

[21] M. A. Therib, H. A. Marzog, and M. J. Mohsin, "Smart digital bi-directional visitors counter based on IoT," Journal of Physics: Conference Series, vol. 1530, pp. 1-7, 2020, doi: 10.1088/1742-6596/1530/1/012018.

[22] M. M. A. Zahra, M. J. Mohsin, and L. A. Abdul-Rahaim, "Artificial intelligent smart home automation with secured camera management-based GSM, cloud computing and Arduino," Periodicals of Engineering and Natural Science, vol. 8, no. 4, pp. 2160-2168, 2020, doi: 10.21533/pen.v8i4.1718.

[23] A. N. Hassan, M. J. Mohsin, and A. H. K. Khwayyir, "Discrimination of ECG signal based on S-interpolation and quantum neural network," Annals of Tropical Medicine and Public Health, vol. 23, no. 10, 2020, doi: 10.36295/ASRO.2020.231039.

[24] H. A. Marzog, M. J. Mohsin, and M. A. Therib, "Chaotic systems with pseudorandom number generate to protect the transmitted data of wireless network," Indonesian Journal of Electrical Engineering and Computer Science (IJECE), vol. 21, no. 3, pp. 1602-1610, Mar. 2021, doi: 10.11591/ijeecs.v21.i3.pp1602-1610.

[25] M. J. Mohsin, H. A. Marzog, and M. A. Therib, "Enhancement throughput and increase security of image transmitted over wireless network using (DNC)," IOP Conf. Series: Materials Science and Engineering: 2nd International Scientific Conference of Al-Ayen University (ISCAU-2020), vol. 928, no. 2, 2020, Art. No. 022078, doi: 10.1088/1757-899X/928/2/022078

\section{BIOGRAPHIES OF AUTHORS}

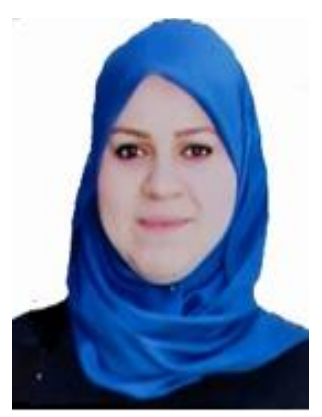

Marwa Jaleel Mohsin (D) S SC P received master's degree in communication engineering, University of Technology/Electrical Engineering Department-Baghdad in 2015. She is a Ph.D. candidate in communication and electronic engineering, University of Babylon/electrical engineering department. She is an instructor in the engineering technical college of Najaf, Al-Furat Al-Awsat Technical University from 2007 till now. The interest in image processing, digital communication, wireless communication, optical communication and digital signal processing. She can be contacted at email: marwa.jaleel@atu.edu.iq.

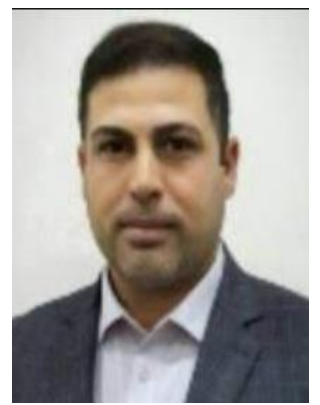

Ibrahim A. Murdas (D) SC P obtained his Ph.D. degree in optical communication engineering, Al Nahrain University in 2005 and professor degree in 2016. He is with the department of electrical engineering, University of Babylon, Hillah, Iraq. His interest in communication systems, digital signal processing, optical communication and optical fibers. He can be contacted at email: eng.ibraheem.abdullaa@uobabylon.edu.iq. 